$\Rightarrow$

\title{
EXTENSION OF THE FOKKER-PLANCK EQUATION
}

\author{
BY \\ JOHN C. PRICE
}

GPO PRICE

$\$$

CFSTI PRICE(S) \$

Hard $(\cdots, \|, C)$

1.00

Microf as (NiF)

50

H 653 Juiros
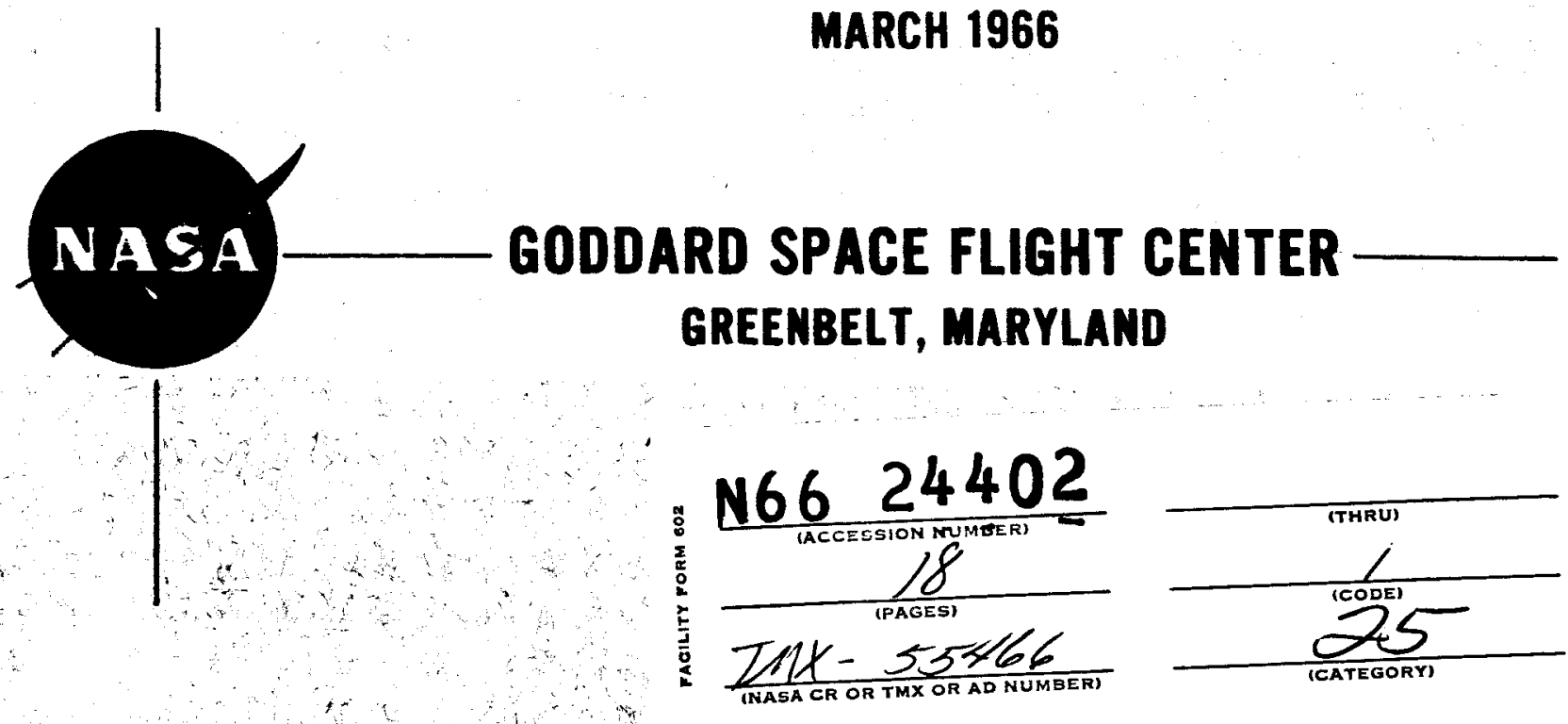


\section{EXTENSION OF THE FOKKER-PLANCK EQUATION}

by

John C. Price*

Lawrence Radiation Laboratory

Berkeley, California

\footnotetext{
*Present address: Laboratory for Theoretical Studies NASA-Goddard Space
} Flight Center, Greenbelt, Maryland. 


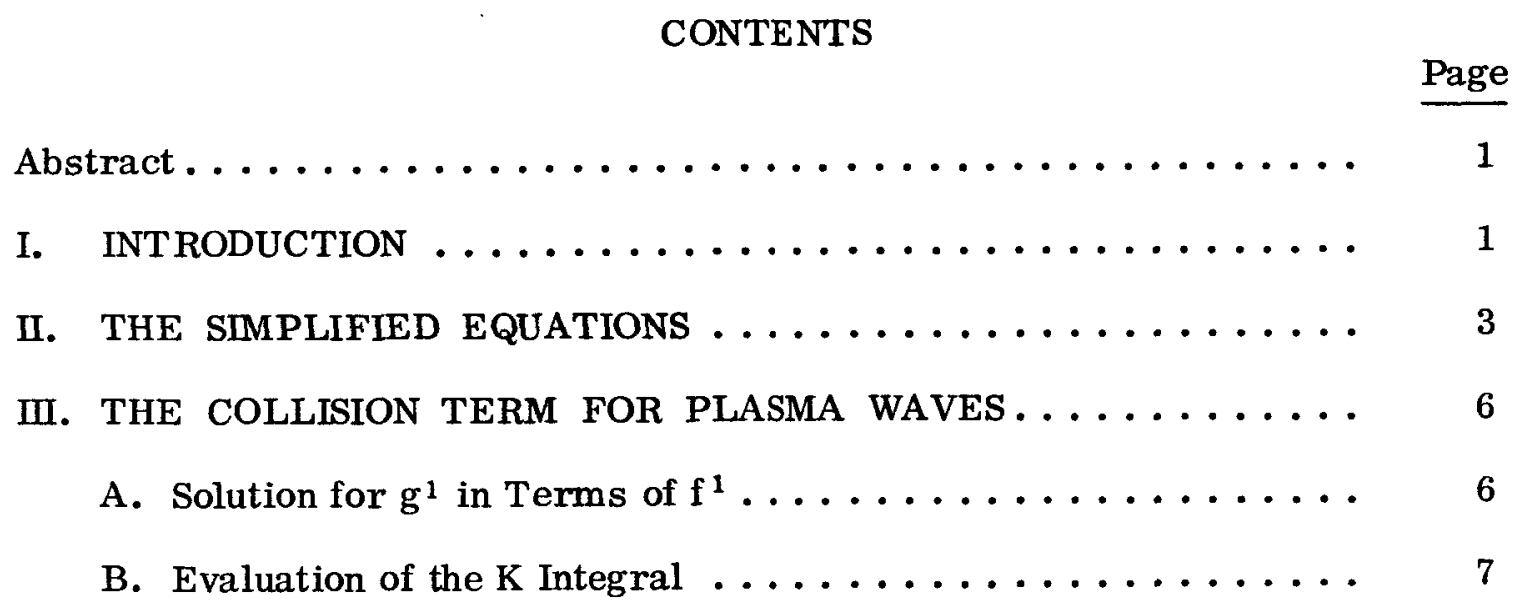

IV. CONCLUSION ...................... 10

Acknowledgment $\ldots \ldots \ldots \ldots \ldots \ldots \ldots \ldots \ldots \ldots \ldots \ldots \ldots \ldots$ 


\title{
EXTENSION OF THE FOKKER-PLANCK \\ EQUATION
}

by

John C. Price

\begin{abstract}
24402

A simple collision term valid to order $1 / \ln \Lambda$ is derived for small amplitude waves in a uniform plasma. This result extends the validity of Fokker-Planck type equations from the domain $0 \leq \omega<\omega_{\mathrm{p}}, 0 \leq \mathrm{k}<<\mathrm{k}_{\mathrm{d}}$ to the domain $0 \leq \omega<\Lambda \omega_{\mathrm{p}}, 0 \leq \mathrm{k} \approx \mathrm{k}_{\mathrm{d}}$.

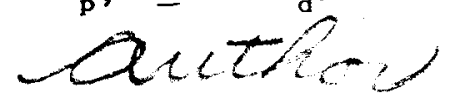

\section{INTRODUCTION}

It is of general interest to obtain the effects of collisions on the behavior of waves in a plasma. Previous efforts have been directed along two main lines. Some authors ${ }^{1,2}$ use the linearized version of the Fokker-Planck equation ${ }^{3}$ to study the effects of collisions on waves. This is certainly correct for the study of low frequency $\left(\omega \sim \nu_{\text {col1ision }}\right)$ long wavelength $\left(\lambda \sim \lambda_{\text {mean free path }}\right)$ waves, but it is not clear how far outside this domain the equation may be trusted. For example it is obviously suspect at frequencies of the order of the plasma frequency.

1. D. A. Tidman, Phys. of Fluids 7, 1826, 1964.

2. G. G. Comisar, Phys. of Fluids $\underline{6}, 76,1963$.

3. M. Rosenbluth, W. M. MacDonald, and D. L. Judd, Phys. Rev. 107, 1, 1957. 
Other authors have used collision models ${ }^{4,5}$ to study the desired effects. These models are constructed to make the resulting analysis relatively tractable, but they are somewhat arbitrary, for they are not based on the reduction of a more exact frequency dependent collision term.

In this paper we shall construct a reasonably accurate collision term for plasma waves. This collision term should prove useful in several respects: for direct analysis of the resulting equation for the full range of collisional effects; for perturbation solutions leading to a relatively simple dispersion relation for waves; and as a valid basis for the construction of model collision terms.

Earlier attempts by Frieman, ${ }^{6}$ Berk, ${ }^{7}$ and Silin ${ }^{8}$ have involved restrictions which will not apply here. Guernseys' result $^{9}$ presumably includes the result to be derived here (except that Guernsey considers waves in an equilibrium plasma), but his analysis is difficult and his result so complicated that the present simpler (and less accurate) derivation seems worthwhile.

In section II the equations of the BBGKY hierarchy are simplified to form the basis of the analysis. In section III the collision term is calculated and the kinetic equation is obtained. In section IV we discuss the validity of the collision term for plasma waves.

4. E. P. Gross and M. Krook, Phys. Rev. 102, 593, 1956.

5. R. L. Liboff, Phys. of Fluids, 5, 963, 1962 .

6. E. Frieman, Journal of Math. Physics, $4,410,1963$.

7. H. Berk, Phys. of Fluids, 17, 257, 1964.

8. V. Silin, JETP, 11, 1277, 1960.

9. R. Guernsey, Phys of Fluids, 5, 322, 1962. 


\section{THE SIMPLIFIED EQUATIONS}

Wo berin with the usual equations of the BBGKY ${ }^{10}$ for the one particle dis-

tribution $f$ and the pair correlation function $g$. With $\left\{\mathbf{r}_{1}, \mathbf{v}_{1}\right\}=\mathrm{X}_{1}$, etc, we have

$$
\begin{aligned}
\left(\frac{\partial}{\partial t}+v_{1} \cdot \nabla_{1}\right) f\left(X_{1}, t\right)-\frac{n}{m} & \frac{\partial f}{\partial \mathbf{v}_{1}} \cdot \int \mathrm{dX} \nabla_{2} \nabla_{1} \frac{\mathbf{q}^{2}}{\left|\mathbf{r}_{1}-\mathbf{r}_{2}\right|} \mathrm{f}\left(\mathrm{X}_{2}, \mathrm{t}\right) \\
& =\frac{\mathrm{n}}{\mathrm{m}} \frac{\partial}{\partial \mathbf{v}_{1}} \cdot \int \mathrm{d} \mathrm{X}_{2} \nabla_{1} \frac{\mathrm{q}^{2}}{\left|\mathbf{r}_{1}-\mathbf{r}_{2}\right|} \mathrm{g}\left(\mathrm{X}_{1}, \mathrm{X}_{2}, \mathrm{t}\right)
\end{aligned}
$$

$$
\begin{aligned}
\left(\frac{\partial}{\partial t}+v_{1} \cdot \nabla_{1}+v_{2} \cdot \nabla_{2}\right) g\left(X_{1}, X_{2}, t\right)=\frac{1}{m} \nabla_{1} \frac{q^{2}}{\left|\mathbf{r}_{1}-\mathbf{r}_{2}\right|} \cdot\left(\frac{\partial}{\partial \mathbf{v}_{1}}-\frac{\partial}{\partial \mathbf{v}_{2}}\right) \\
\quad \times f\left(X_{1}, t\right) f\left(X_{2}, t\right)+\frac{n}{m}\left[\int d X_{3} f\left(X_{3}, t\right) \nabla_{1} \frac{q^{2}}{\left|r_{1}-r_{3}\right|} \cdot \frac{\partial}{\partial \mathbf{v}_{1}}+(\mapsto-2)\right] \\
\quad \times g\left(X_{1}, X_{2}, t\right)+\frac{n}{m}\left[\int d X_{3} \frac{\partial f\left(X_{1}, t\right)}{\partial v_{1}} \cdot \nabla_{1} \frac{q^{2}}{\left|\mathbf{r}_{1}-\mathbf{r}_{3}\right|} g\left(X_{2}, X_{3}, t\right)\right.
\end{aligned}
$$$$
+(1-2)]
$$

In equation 2 the terms involving three particle correlations have been neglected, as well as the terms involving $g$ which are significant primarily when $\left|\mathbf{r}_{1}-\mathbf{r}_{2}\right|$ is small. The neglect of these latter terms forces the usual short range cutoff in the $r_{2}$ integration in 1 .

10. D. C. Montgomery and D. A. Tidman, Plasma Kinetic Theory, (McGraw-Hill, New York, 1964), p. 44. 
The neglect of the term in $g$ of equation 1 leads to the familiar Vlasov equation; it is this "small" term which we wish to calculate. To do this we must solve 2 to find $g$ in terms of $f$, then insert the result in 1 . This problem (in the linearized approximation, which we shall use here) has been solved by Guernsey, but the analysis and result are formidable. We shall obtain a crude (order $1 / \ln \Lambda$ ) result after making further approximations on equation 2 .

We first estimate the magnitude of the various terms of 2 , then neglect the less important ones. Since we do not commit outselves to a value for the wave frequency the term $\partial / \partial \mathrm{t}$ may be large or small-in general we must keep it. We estimate velocities and velocity gradients by the thermal velocity $\mathrm{V}_{\mathrm{th}}$. Because $\int \mathrm{fdv}=1$, we take $\mathrm{f}=1 / \mathrm{V}_{\mathrm{th}}^{3}$ in a volume $\mathrm{V}_{\mathrm{th}}^{3}$. A more exact analysis shows that two particle correlations are cutoff (decrease rapidly) for $\left|\mathbf{r}_{1}-\mathbf{r}_{2}\right|$ greater than the Debye length $\lambda_{d}$. Thus we estimate distances for $g$ by a length $L$ where $\lambda_{d}>L>r_{0}$, with $r_{0}$ the closest approach distance. Of course $g$ also varies due to the variation of $f$. Thus we must have $\mathrm{L}$ much less than the scale length of $\mathrm{f}$, which restricts our analysis to waves with a wavelength of the order of or greater than $\lambda_{d}$.

Inserting these estimates in 2 , we find

$$
\begin{aligned}
\left(\frac{\partial}{\partial \mathrm{t}}: \frac{\mathrm{V}_{\mathrm{th}}}{\mathrm{L}}:\right. & \left.\frac{\mathrm{V}_{\mathrm{th}}}{\mathrm{L}}\right)_{\mathrm{g}}=\frac{1}{\mathrm{~m}} \frac{\mathrm{q}^{2}}{\mathrm{~L}^{2}}\left(\frac{1}{\mathrm{~V}_{\mathrm{th}}}: \frac{1}{\mathrm{~V}_{\mathrm{th}}}\right) \frac{1}{\mathrm{~V}_{\mathrm{th}}^{3}} \frac{1}{\mathrm{~V}_{\mathrm{th}}^{3}} \\
& : \frac{\mathrm{n}}{\mathrm{m}}\left[\frac{\mathrm{V}_{\mathrm{th}}^{3} \mathrm{~L}^{3}}{\mathrm{~V}_{\mathrm{th}}^{3}} \frac{\mathrm{q}^{2}}{\mathrm{~L}^{2}} \frac{1}{V_{\mathrm{th}}}\right]:(1-2) \mathrm{g}: \frac{\mathrm{n}}{\mathrm{m}}\left[\frac{1}{\mathrm{~V}_{\mathrm{th}}^{4}} \mathrm{~V}_{\mathrm{th}}^{3} \mathrm{~L}^{3} \frac{\mathrm{q}^{2}}{\mathrm{~L}^{2}} \mathrm{~g}:(1-2)\right](3)
\end{aligned}
$$


We collect and cancel factors, and use the fact that

$$
\frac{n q^{2}}{m} \approx \omega_{p}^{2} \approx \frac{v_{t h}^{2}}{\lambda_{d}^{2}}
$$

to find

$$
\begin{array}{r}
\left(\frac{\partial}{\partial t}: \frac{V_{t h}}{L}: \frac{V_{t h}}{L}\right) g=\frac{1}{m} \frac{q^{2}}{L^{2}}\left(\frac{1}{V_{t h}^{7}}: \frac{1}{V_{t h}^{7}}\right):\left[\frac{V_{t h}}{L}\left(\frac{L}{\lambda_{d}}\right)^{2}+(1-2)\right] g \\
:\left[\frac{V_{t h}}{L}\left(\frac{L}{\lambda d}\right)^{2}+(1 \leftrightarrow 2)\right] g
\end{array}
$$

Since $L<\lambda_{d}$ we may drop the right hand terms in $g$ compared to those on the left. The resulting equation for $\mathrm{g}$ is sometimes called the weak coupling equation. ${ }^{6}$

$\left(\frac{\partial}{\partial t}+\mathbf{v}_{1} \cdot \nabla_{1}+\mathbf{v}_{2} \cdot \nabla_{2}\right) g=\frac{1}{m} \nabla_{1} \frac{q^{2}}{\left|\mathbf{r}_{1}-\mathbf{r}_{2}\right|} \cdot\left(\frac{\partial}{\partial \mathbf{v}_{1}}-\frac{\partial}{\partial \mathbf{v}_{2}}\right) \mathrm{f}\left(\mathrm{X}_{1}, \mathrm{t}\right) \mathrm{f}\left(\mathrm{X}_{2}, \mathrm{t}\right)(5)$

As a result of these approximations we must supply both long and short range cutoffs to the $r_{2}$ integration in equation 1 . The resulting collision term will be valid to order $1 / \ln \Lambda$.

We now linearize $f$ and $g$ about a uniform stationary state: $f=f^{0}\left(v_{1}\right)+f^{1}\left(r_{1}\right.$, $\left.v_{1}, t\right) ; g=g^{0}\left(\left|r_{1}-r_{2}\right|, v_{1}, v_{2}\right)+g^{1}\left(r_{1}, r_{2}, v_{1}, v_{2}, t\right)$. We treat superscript one terms as small, and discard products of such terms. The neglect of time dependence for $\mathrm{f}^{0}$ and $\mathrm{g}^{0}$ implies that then actual time dependence should be slower than that of $f^{1}$ and $g^{1}$. Thus for very low frequency waves $f^{0}$ must be

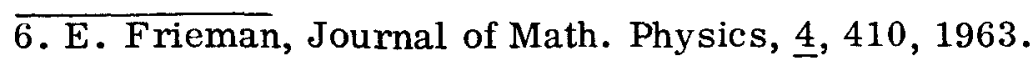


the equilibrium (Maxwellian) distribution. For waves below the collision frequency $\nu_{c}$, the linearized theory is inappropriate and the nonlinear FokkerPlanck equation must be used. In keeping with the notation of the Vlasov equation we set $E^{1}=-n q \int d X_{2} \nabla_{1} 1 /\left|r_{1}-r_{2}\right| f^{1}\left(X_{2}, t\right)$, and find the equations for $\mathrm{f}^{1}$ and $\mathrm{g}^{1}$

$\left(\frac{\partial}{\partial t}+v_{1} \cdot \nabla_{1}\right) f^{1}\left(X_{1}, t\right)+\frac{q}{m} E^{1}\left(r_{1}, t\right) \cdot \frac{\partial f^{0}}{\partial v_{1}}=\frac{n}{m} \frac{\partial}{\partial v_{1}} \cdot \int d X_{2} \nabla_{1} \frac{q^{2}}{\left|r_{1}-r_{2}\right|} g^{1}$

$\left(\frac{\partial}{\partial t}+\mathbf{v}_{1} \cdot \nabla_{1}+\mathbf{v}_{2} \cdot \nabla_{2}\right) \mathrm{g}^{1}\left(\mathrm{X}_{1}, \mathrm{X}_{2}, \mathrm{t}\right)=\frac{1}{\mathrm{~m}} \nabla_{1} \frac{\mathrm{q}^{2}}{\left|\mathbf{r}_{1}-\mathbf{r}_{2}\right|} \cdot\left(\frac{\partial}{\partial \mathbf{v}_{1}}-\frac{\partial}{\partial \mathbf{v}_{2}}\right)$

$$
x\left[f^{1}\left(x_{1}, t\right) f^{0}\left(v_{2}\right)+f^{0}\left(v_{1}\right) f^{1}\left(x_{2}, t\right)\right]
$$

III. THE COLLISION TERM FOR PLASMA WAVES

\section{A. SOLUTION FOR $\mathrm{g}^{1}$ IN TERMS OF $\mathrm{f}^{1}$}

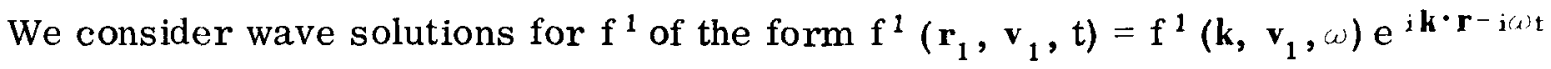
where $\omega$ is in the upper half $\omega$ plane, or else analytically continued ${ }^{11}$ into the lower half plane. Equation 6 may be written

$$
\begin{aligned}
& \left(-\mathrm{i} \omega+\mathbf{i} \mathbf{k} \cdot \mathbf{v}_{1}\right) \mathrm{f}^{1}\left(\mathbf{k}, \mathbf{v}_{1}, \omega\right)+\frac{\mathrm{q}}{\mathrm{m}} \mathrm{E}^{1}(\mathbf{k}, \omega) \cdot \frac{\partial \mathrm{f}^{0}}{\partial \mathbf{v}_{1}}= \\
& \begin{aligned}
\frac{\mathrm{nq}}{\mathrm{m}} \frac{\partial}{\partial \mathbf{v}_{1}} \cdot \int \mathrm{d} \mathbf{v}_{2} \int \frac{\mathrm{d} \mathbf{K}}{2 \pi^{2}} \frac{\mathrm{iK}}{\mathrm{k}^{2}} \mathrm{~g}^{1}\left(\mathbf{k}-\mathbf{K}, \mathbf{K}, \mathbf{v}_{1}, \mathbf{v}_{2}, \omega\right) \\
=\frac{\partial}{\partial \mathbf{v}_{1}} \cdot \mathbf{J}\left(\mathbf{k}, \mathbf{v}_{1}, \omega\right)
\end{aligned}
\end{aligned}
$$

$\overline{11 . ~ L . ~ L a n d a u, ~ J . ~ P h y s . ~(U . S . S . R .) ~} \underline{10}, 25,1946$. 
Appropriate Fourier analysis of 7 yields

$$
\begin{aligned}
& \left(-\mathrm{i} \omega+\mathrm{i}(\mathbf{k}-\mathbf{K}) \cdot \mathbf{v}_{1}+\mathrm{iK} \cdot \mathbf{v}_{2}\right) \mathrm{g}^{1}\left(\mathbf{k}-\mathbf{K}, \mathbf{K}, \mathbf{v}_{1}, \mathbf{v}_{2}, \omega\right) \\
& =-\frac{4 \pi \mathrm{iq} \mathbf{q}^{2}}{\mathrm{~m}}\left(\frac{\partial}{\partial \mathbf{v}_{1}}-\frac{\partial}{\partial \mathbf{v}_{2}}\right) \cdot\left[\frac{\mathbf{K}}{\mathrm{K}^{2}} \mathrm{f}^{1}\left(\mathbf{k}, \mathbf{v}_{1}, \omega\right) \mathrm{f}^{0}\left(\mathbf{v}_{2}\right)+\frac{\mathbf{K}-\mathbf{k}}{(\mathrm{K}-\overline{\mathrm{k}})^{2}}\right. \\
& \left.\times \mathrm{f}^{0}\left(\mathbf{v}_{1}\right) \mathrm{f}^{1}\left(\mathbf{k}, \mathbf{v}_{2}, \omega\right)\right]
\end{aligned}
$$

We insert the result for $\mathrm{g}^{1}$ into 8 and find

$$
\begin{aligned}
& \mathrm{J}\left(\mathbf{k}, \mathbf{v}_{1}, \omega\right)=\frac{2 \mathrm{nq}}{\pi \mathrm{m}^{2}} \int \mathrm{d} \mathbf{v}_{2} \int \frac{\mathrm{dKK}}{\mathrm{K}^{2}} \frac{1}{-i \omega+i k \cdot \mathbf{v}_{1}+\mathrm{iK} \cdot\left(\mathbf{v}_{1}-\mathbf{v}_{2}\right)} \\
& \times\left(\frac{\partial}{\partial \mathbf{v}_{1}}-\frac{\partial}{\partial \mathbf{v}_{2}}\right) \cdot\left[\frac{\mathrm{K}}{\mathrm{K}^{2}} \mathbf{f}^{1}\left(\mathbf{k}, \mathbf{v}_{1}, \omega\right) \mathrm{f}^{0}\left(\mathbf{v}_{2}\right)+\frac{\mathrm{K}-\mathbf{k}}{(\mathrm{K}-\mathrm{k})^{2}} \mathrm{f}^{0}\left(\mathbf{v}_{1}\right) \mathrm{f}^{1}\left(\mathbf{k}, \mathbf{v}_{2}, \omega\right)\right](10)
\end{aligned}
$$

\section{B. EVALUATION OF THE K INTEGRAL}

In order to evaluate the $K$ integral in (10) we apply the Plemelj formula ${ }^{12}$ to the quantity $\left[\omega-\mathbf{k} \cdot \mathbf{v}_{1}+\mathbf{K} \cdot\left(\mathbf{v}_{1}-\mathbf{v}_{2}\right)\right]^{-1}$, noting that $\omega$ is analytically continued from the upper half plane.

$\frac{1}{\omega-\mathbf{k} \cdot \mathbf{v}_{1}+K \cdot\left(\mathbf{v}_{1}-\mathbf{v}_{2}\right)}=\frac{\mathbf{P}}{\omega-\mathbf{k} \cdot \mathbf{v}_{1}+K \cdot\left(\mathbf{v}_{1}-\mathbf{v}_{2}\right)}-\mathbf{i} \pi \delta\left[\omega-\mathbf{k} \cdot \mathbf{v}_{1}+K \cdot\left(\mathbf{v}_{1}-\mathbf{v}_{2}\right)\right]$

For $\omega \ll \mathrm{k}_{0} \mathrm{~V}_{\mathrm{th}} \approx \Lambda \omega_{\mathrm{p}}$ the principal part is odd for large $\mathrm{K}$, and the resulting integral may be neglected compared to that over the delta function.

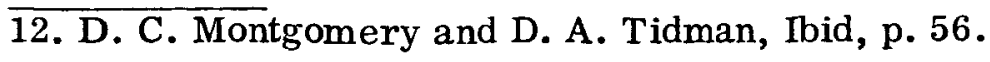


The order $1 / \ln \Lambda$ error is comparable to that caused by the arbitrary cutoff.

The resulting collision term may be written

$$
\begin{array}{rl}
J\left(k, v_{1}, \omega\right)=\frac{2 \pi n q^{4}}{m^{2}} \int d \mathbf{v}_{2} & Q\left(\omega-\mathbf{k} \cdot \mathbf{v} ; \mathbf{v}_{1}-\mathbf{v}_{2}\right) \cdot\left(\frac{\partial}{\partial \mathbf{v}_{1}}-\frac{\partial}{\partial \mathbf{v}_{2}}\right) \\
\times & {\left[\mathrm{f}^{1}\left(\mathbf{k}, \mathbf{v}_{1}, \omega\right) \mathrm{f}^{0}\left(\mathbf{v}_{2}\right)+\mathrm{f}^{0}\left(\mathbf{v}_{1}\right) \mathrm{f}^{1}\left(\mathbf{k}, \mathbf{v}_{2}, \omega\right)\right]}
\end{array}
$$

where $Q$ is given by

$$
Q(\mathbf{a} ; \mathbf{b})=\frac{1}{\pi} \int_{|\mathbf{K}|=\mathrm{k}_{\mathrm{d}}=2 \pi / \lambda d}^{|\mathbf{K}|=\mathrm{k}_{0}=2 \pi / \mathrm{r}_{0}} \frac{\mathbf{K K}}{\mathrm{K}^{4}} \delta(\mathbf{a}-\mathbf{K} \cdot \mathbf{b}) \mathrm{dK}
$$

Here we have neglected $\mathbf{k}$ in the factor $(\mathbf{K}-\mathbf{k}) /(\mathrm{K}-\mathbf{k})^{2}$ of equation 10 . We could also displace the origin of the $\mathrm{K}$ integral over this factor by $k$, then drop k. This leads to a symmetric collision term, but the difference from (12) is not significant to within the accuracy of the expression.

To evaluate $Q$ we observe that the only vector available in $(13)$ is $\mathbf{b}$, so Q must have the form

$$
\mathbf{Q}=\mathbf{A I}+\mathbf{B b b}
$$


where $I$ is the unit tensor. We $\operatorname{dot}(13)$ and 14 twice with b to produce

$$
\begin{aligned}
\mathrm{Ab}^{2}+\mathrm{Bb}^{4} & =\frac{1}{\pi} \int \frac{\mathrm{dK}}{\mathrm{K}^{4}}(\mathbf{K} \cdot \mathbf{b})^{2} \delta(\mathbf{a}-\mathbf{K} \cdot \mathbf{b}) \\
& \approx 0
\end{aligned}
$$

Again the error is order $1 / \ln \Lambda$ compared to the term we keep. Using (15) $\left(B=-A / b^{2}\right)$, and taking the trace of (13) and (14), we find

$$
A=\frac{1}{2 \pi} \int_{|\mathbf{K}|=\mathbf{k}_{d}}^{|\mathbf{K}|=\mathrm{k}_{0}} \frac{\mathrm{d} \mathbf{K}}{\mathrm{K}^{2}} \delta(a-K \cdot \mathbf{b})
$$

The integral is elementary when expressed in cylindrical coordinates with axis parallel to $b$. We find for $Q$

$$
\begin{aligned}
Q(\mathbf{a} ; \mathbf{b}) & =\ln \Lambda\left(\frac{\mathrm{b}^{2} \mathbf{I}-\mathbf{b b}}{\mathrm{b}^{3}}\right) \quad \text { for } \quad \frac{a}{b}<k_{d} \\
& =\ln \frac{k_{0} b}{a}\left(\frac{b^{2} \mathbf{I}-\mathbf{b b}}{b^{3}}\right) \quad \text { for } \quad k_{d}<\frac{a}{b}<k_{0} \\
& =0 \quad \text { for } \quad k_{0}<\frac{a}{b}
\end{aligned}
$$

The generalization from single species to a multiple species plasma is straightforward. We use Greek subscripts to indicate particle species, and 
combine 8, 12 and 17 to find the kinetic equation for species $\mu$.

$$
\begin{gathered}
\left(-\mathbf{i} \omega+\mathbf{i} \mathbf{k} \cdot \mathbf{v}_{1}\right) \mathbf{f}_{\mu}{ }^{1}\left(\mathbf{k}, \mathbf{v}_{1}, \omega\right)+\frac{\mathrm{q}_{\mu}}{\mathrm{m}_{\mu}} \mathrm{E}^{1}(\mathbf{k}, \omega) \cdot \frac{\partial \mathbf{f}_{\mu}{ }^{0}}{\partial \mathbf{v}_{1}}=\frac{2 \pi \mathrm{q} \mu^{2}}{\mathrm{~m}_{\mu}} \\
\times \sum_{\nu} \mathrm{n}_{\nu} \mathrm{q}_{\nu}{ }^{2} \frac{\partial}{\partial \mathbf{v}_{1}} \cdot \int \mathrm{d} \mathbf{v}_{2} \mathrm{Q}\left(\omega-\mathbf{k} \cdot \mathbf{v}_{1} ; \mathbf{v}_{1}-\mathbf{v}_{2}\right) \cdot\left(\frac{1}{\mathrm{~m}_{\mu}} \frac{\partial}{\partial \mathbf{v}_{1}}-\frac{1}{\mathrm{~m}_{\nu}} \frac{\partial}{\partial \mathbf{v}_{2}}\right) \\
\times\left[\mathrm{f}_{\nu}{ }^{1}\left(\mathbf{k}, \mathbf{v}_{1}, \omega\right) \mathrm{f}_{\nu}{ }^{2}\left(\mathbf{v}_{2}\right)+\mathbf{f}_{\nu}{ }^{1}\left(\mathbf{k}, \mathbf{v}_{2}, \omega\right) \mathbf{f}_{\mu}{ }^{0}\left(\mathbf{v}_{1}\right)\right]
\end{gathered}
$$

\section{CONCLUSION}

It is probably not necessary to emphasize the fact that the collision term of equation 18 is approximate. Clearly "order $1 / \ln \Lambda^{\prime \prime}$ is a better expression for the error than is " $1 / \ln \Lambda "$. On the other hand even the thorough solution of the problem by Guernsey suffers the defect of the short range (large K) divergence, so that the more complicated result is not formally better than that given here. The collision term of (18) has the virtue of simplicity.

This collision term differs from the usual Fokker-Planck term ${ }^{3}$ in that the coulomb logarithm has frequency and wavenumber dependence. This dependence involves both the relative velocity of the particles $\left|v_{1}-v_{2}\right|$ and the doppler shifted frequency $|\omega-\mathbf{k} \cdot \mathbf{v}|$. We establish a rough criterion for the significance of this dependence by requiring that most particles $|\mathbf{v}|<V_{t h}$ be within the region where the logarithm is decreased for $\ln \Lambda$. This yields $\omega \approx \mathrm{kd} \mathrm{V}_{\mathrm{th}} \approx \omega_{\mathrm{p}}$. Due to the slow (logarithmic) variation we may state that the Fokker-Planck collision term is modified significantly at frequencies above the plasma frequency.

3. M. Rosenbluth, W. M. MacDonald, and D. L. Judd, Phys. Rev. 107, 1, 1957. 


\section{Acknowledgment}

The author wishes to thank Dr. Allan Yaufman for discussions concerning this work. The work was performed under the auspices of the Atomic Energy Commission. 